\section{Method for optimizing methylation-specific PCR}

\author{
Eric Smith¹, Tina Bianco-Miotto ${ }^{1}$, Paul Drew ${ }^{2}$, and David Watson ${ }^{2}$ \\ ${ }^{1}$ The University of Adelaide, ${ }^{2}$ Flinders University, Adelaide, Australia
}

BioTechniques 35:32-33 (July 2003)
The methylation of the $\mathrm{CpG}$ islands that are associated with the promoters of numerous genes can be associated with gene silencing. Therefore, it is an important area of research, and several techniques have been developed for the rapid and sensitive analysis of methylation. One of these methods is methylation-specific PCR (1). Most commonly, the PCR conditions are optimized using DNA prepared from cell lines that have a relatively homogeneous methylation pattern. However, in the typical tissue specimen, there will be a mixture of cells, with a number of patterns of methylation. We report that the optimization steps, which are appropriate when working with DNA prepared from cell populations with uniform methylation patterns, are not appropriate when analyzing DNA from tissues in which the subsets of cells have different methylation patterns.

Common to the PCR-based methods to detect methylation is a bisulfite modification step that creates sequence differences in the DNA. Unmethylated cytosines of a $\mathrm{CpG}$ dinucleotide are converted to uracils, while methylated cytosines of a $\mathrm{CpG}$ dinucleotide are protected from change (2). In methylation-specific PCR, the sequence differences are detected by amplification using primers that distinguish between the methylated and unmethylated bisulfite-modified DNA and unmodified DNA. In the design of primers specific for methylated DNA, cytosines that are conserved because of their methylation are placed in the $3^{\prime}$ end. In the design of primers that will only amplify unmethylated DNA after bisulfite modification, thymidines, derived from converted cytosines, are placed in the $3^{\prime}$ end. Stringency is increased with their positioning as close as possible to the $3^{\prime}$ end of the primer. A disadvantage of methylation-specific PCR is that only the few $\mathrm{CpG}$ sites that are situated within the template sequence to which the primers bind can be investigated for a given primer pair.

We have used methylation-specific PCR to study the methylation status of genes in premalignant and malignant esophageal tissue. In premalignant tissue in particular, the cells of interest may be present in relatively low number, making it necessary to use an assay able to detect methylated sequences present in a relatively low concentration relative to the unmethylated sequence. We have found that the apparent sensitivity of methylation-specific PCR varies depending on the method for determining the optimal annealing temperatures to use in the PCR, particularly when testing mixtures of methylated and unmethylated DNA. Here we describe an efficient optimization method that results in increased sensitivity in these situations.

We prepared DNA from cells in which the promoter of the gene of interest was known to be methylated and from cells known to be unmethylated. The DNA was bisulfite-modified using the method described by Paulin et al. (3). Two micrograms of genomic DNA in $18 \mu \mathrm{L}$ water were denatured by adding $2 \mu \mathrm{L}$ freshly made $3 \mathrm{M} \mathrm{NaOH}$ followed by incubation at $37^{\circ} \mathrm{C}$ for 15 min. Then, $280 \mu \mathrm{L}$ of a $6.24 \mathrm{M}$ urea/4 $\mathrm{M}$ bisulfite solution and $2 \mu \mathrm{L} 100 \mathrm{mM}$ hydroquinone were added. The samples were then incubated for 16 cycles of $55^{\circ} \mathrm{C}$ for $15 \mathrm{~min}$ and $95^{\circ} \mathrm{C}$ for $30 \mathrm{~s}$. The modified DNA was purified using the Concert ${ }^{\mathrm{TM}}$ Rapid PCR Purification System (Invitrogen, Sydney, NSW, Australia), eluting with $50 \mu \mathrm{L}$ TE buffer. In the optimization and running of the methylation-specific PCR, PCR was carried out for 35 cycles in a Mastercycler ${ }^{\circledR}$ gradient (Eppendorf South Pacific Pty. Ltd., North Ryde, NSW, Australia), with each $25 \mu \mathrm{L}$ reaction containing $2 \mu \mathrm{L}$ target DNA, $0.2 \mathrm{mM}$ dNTPs, $0.2 \mu \mathrm{M}$ each primer, $2 \mathrm{mM}$ $\mathrm{MgCl}_{2}$, and $1 \mathrm{U}$ HotStarTaq ${ }^{\mathrm{TM}}$ DNA polymerase in $1 \times$ PCR buffer (Qiagen, Clifton Hill, VIC, Australia).

Conventionally, the optimal annealing temperature for a methylation-specific PCR primer pair is determined empirically from temperature gradients performed separately on the bisulfitemodified methylated DNA, bisulfitemodified unmethylated DNA, and the

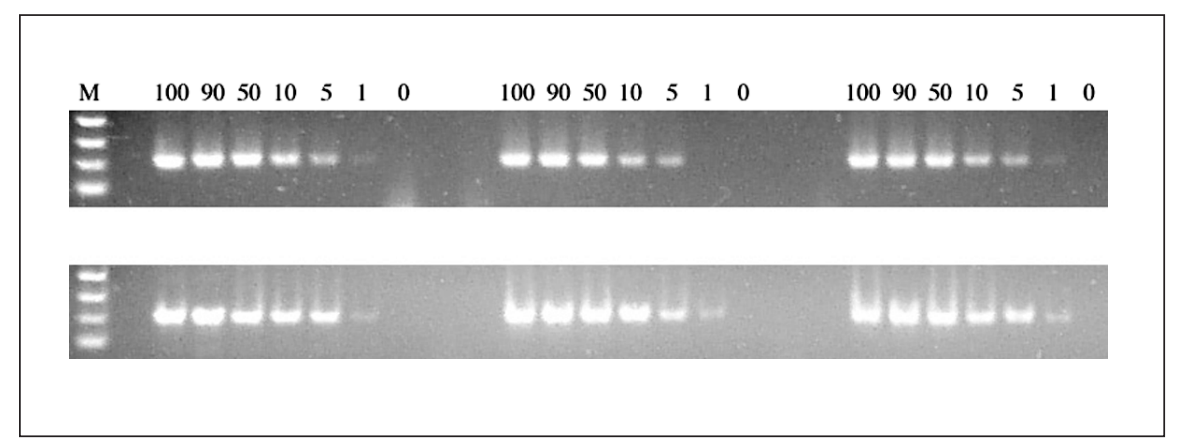

Figure 1. Gradient PCR using a primer pair specific for the methylated p16 promoter. The temperatures of the reactions in each gel from left to right were $60.5^{\circ}, 62.2^{\circ}$, and $63.7^{\circ} \mathrm{C}$. Top: Bisulfitemodified methylated DNA diluted in unmethylated DNA (lanes from left to right: 100:0, 90:10, 50:50, 10:90, 5:95, 1:99, and 0:100) was added to the PCR. Bottom: The same amount of modified methylated DNA, diluted into water (lanes from left to right: $100 \%, 90 \%, 50 \%, 10 \%, 5 \%, 1 \%$, and $0 \%$ ), was added. (Forward methylated specific p16 primer: 5'-tTaTTagagggtggggcggaTcgc-3'; and reverse methylated specific p16 primer: 5'-cgacccegAAccgcgAccgtA-3'. Bisulfite-modified cytosines are denoted by a capital $\mathrm{T}$ in the forward primer and a capital $\mathrm{A}$ in the reverse primer. $\mathrm{M}$ denotes the marker.) 

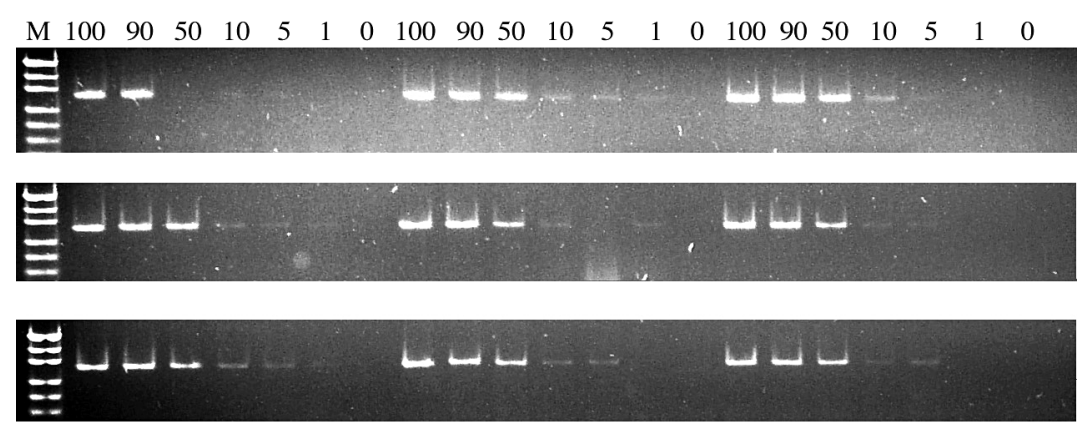

Figure 2. Gradient PCR using a primer pair specific for the unmethylated Cyclin D2 promoter The reaction temperatures from left to right and top to bottom were $53.1^{\circ}, 53.5^{\circ}, 54.4^{\circ}, 55.6^{\circ}, 57.1^{\circ}$, $58.7^{\circ}, 60.3^{\circ}, 61.9^{\circ}$, and $63.3^{\circ} \mathrm{C}$. At each temperature, modified unmethylated DNA diluted into modified methylated DNA (lanes from left to right: 100:0, 90:10, 50:50, 10:90, 5:95, 1:99, and 0:100) was added to the PCR. (Forward unmethylated specific Cyclin D2 primer: 5'-gTatgTgttagagtaTgtgttagggtTgatT-3', and reverse unmethylated specific Cyclin D2 primer: $5^{\prime}$-ctAacaaAccAcccAaAAtAtctAcActAaAcA-3'. Bisulfite-modified cytosines are denoted by a capital $\mathrm{T}$ in the forward primer and a capital $\mathrm{A}$ in the reverse primer. $\mathrm{M}$ denotes the marker.)

corresponding unmodified DNA. These show if there is any temperature at which unmodified DNA is amplified and at which temperatures the primers for methylated DNA do not amplify unmethylated DNA, or vice versa. The lowest temperature that amplifies only the appropriate DNA is then used for the PCR because at the colder annealing temperatures there is greater PCR yield.

To determine the PCR sensitivity, we ran a temperature gradient, with decreasing amounts of target DNA in water added to the reaction mixture (DNA concentration at $100 \%, 90 \%, 50 \%$, $10 \%, 5 \%, 1 \%$, or $0 \%$ ). In the example presented using primers specific for the methylated $\mathrm{p} 16$ gene promoter region, methylated DNA could be detected when present down to a concentration of $1 \%$ of the maximum (Figure 1, bottom gel). The gradient was then run using dilutions of the methylated DNA made in a solution of unmethylated DNA so that the ratios of methylated to unmethylated DNA were 100:0, 90:10, $50: 50,10: 90,5: 95,1: 99$, and 0:100 (Figure 1, top gel). The amount of product, and hence the sensitivity, was significantly reduced (Figure 1; compare the bands at $1 \%-10 \%$ for the methylated DNA diluted in unmethylated DNA, with the same amount of methylated DNA diluted in water). Similar results were obtained with other genes tested.

This led us to devise a new method for optimizing the annealing temperature, in which specificity and optimal temperature were determined in the one-gradient PCR. Serial dilutions of the DNA type of interest were made into a solution of the alternative DNA (e.g., methylated DNA serially diluted into a solution of unmethylated DNA). Controls were the unmodified DNA and the alternative DNA alone. Figure 2 shows the results for the gradient PCR using the primer pair specific for the unmethylated Cyclin D2 promoter with varying ratios of methylated and unmethylated DNA. At low temperatures, methylation-specific PCR lacks sensitivity, only amplifying target DNA when it is present at $90 \%$ of total DNA. However, at higher temperatures, there is amplification when the target DNA is present at $5 \%$ of total DNA.

When temperature gradients were run using varying mixtures of known amounts of methylated and unmethylated DNA, it was found that optimal specificity and sensitivity occurred at higher temperatures than would have been deduced from running one of the forms of DNA as target alone. The reasons for this are not known. Possibly, at lower temperatures there was a degree of binding of the primers to the alternative DNA, although not appropriate binding for extension to occur. The methylation-specific PCR primers were designed to have a $\mathrm{CpG}$ close to the $3^{\prime}$ end to increase specificity, so it is conceivable that irrelevant binding took place between other sequences in common on both the methylated and unmethylated bisulfite-treated DNA but that effective binding could not occur at the $3^{\prime}$ end. The binding would reduce the concentration of primers available but not result in product. Increasing the temperature would result in less irrelevant binding and so increase sensitivity by increasing the amount of primer available to bind to the relevant DNA.

In situations where there is expected to be a mixture of methylated and unmethylated DNA for a gene of interest, as in analyzing DNA prepared from tissues, the PCR parameters should be optimized using a range of mixtures of the two types of DNA. The preferred temperature may be higher than would be assumed from testing the primers against target DNA alone, perhaps because of competition or interference from the alternative DNA.

\section{ACKNOWLEDGMENT}

E.S. and T.B-M. contributed equally to this work.

\section{REFERENCES}

1.Herman, J.G., J.R. Graff, S. Myohanen, B.D. Nelkin, and S.B. Baylin. 1996. Methylation-specific PCR: a novel PCR assay for methylation status of $\mathrm{CpG}$ islands. Proc. Natl. Acad. Sci. USA 93:9821-9826.

2.Frommer, M., L.E. McDonald, D.S. Millar, C.M. Collis, F. Watt, G.W. Grigg, P.L. Molloy, and C.L. Paul. 1992. A genomic sequencing protocol that yields a positive display of 5'-methylcytosine residues in individual DNA strands. Proc. Natl. Acad. Sci. USA 89:18271831

3.Paulin, R., G.W. Grigg, M.W. Davey, and A.A. Piper. 1998. Urea improves efficiency of bisulphite-mediated sequencing of 5'-methylcytosine in genomic DNA. Nucleic Acids Res. 26:5009-5010.

Received 30 September 2002; accepted 7 April 2003

Address correspondence to Paul Drew, School of Nursing and Midwifery, Faculty of Health Sciences, Flinders University, GPO Box 2100, Adelaide 5001, Australia, e-mail:paul.drew@adelaide.edu.au 